\title{
Enhanced Hemocompatibility of Silver Nanoparticles Using the Photocatalytic Properties of Titanium Dioxide
}

\author{
Xiao Chen, Sheng Dai, Luying Liu, Peng Liu, Peng Ye, Yuzhen Liao, Ansha Zhao, Ping Yang *, \\ Nan Huang * and Jiang Chen *
}

Key Laboratory for Advanced Technologies of Materials, Institute of Biomaterials and Surface Engineering, Ministry of Education, Southwest Jiaotong University, Chengdu, China

Silver nanoparticles (AgNPs) are widely used because of their excellent antimicrobial properties. However, the poor hemocompatibility limits the application of AgNPs in blood contact materials. General approaches to improve the hemocompatibility of AgNPs-

OPEN ACCESS

Edited by:

Guicai Li,

Nantong University, China

Reviewed by:

Zhoukun He,

Chengdu University, China

Tao Gong,

University of South China, China

*Correspondence:

Ping Yang

yangping8@263.net

Nan Huang

nhuang@263.net

Jiang Chen

283876533@qq.com

Specialty section:

This article was submitted to Biomaterials,

a section of the journal

Frontiers in Bioengineering and

Biotechnology

Received: 15 January 2022

Accepted: 01 February 2022

Published: 17 February 2022

Citation:

Chen X, Dai S, Liu L, Liu P, Ye P, Liao Y, Zhao $A$, Yang $P$. Huang $N$ and Chen $J$

(2022) Enhanced Hemocompatibility of

Silver Nanoparticles Using the

Photocatalytic Properties of

Titanium Dioxide.

Front. Bioeng. Biotechnol. 10:855471.

doi: 10.3389/fbioe.2022.855471 containing surfaces are to construct barrier layers or co-immobilize anticoagulant biomolecules. But such modification strategies are often cumbersome to prepare and have limited applications. Therefore, this study proposes a simple UV-photofunctionalization strategy to improve the hemocompatibility of AgNPs. We loaded AgNPs onto titanium dioxide $\left(\mathrm{TiO}_{2}\right)$ nanoparticles to form a composite nanoparticles (Ag@TiO ${ }_{2} \mathrm{NPs}$ ). Then, UV treatment was performed to the $\mathrm{Ag} @ \mathrm{TiO}_{2} \mathrm{NPs}$, utilizing the diffusible photo-induced anticoagulant properties of $\mathrm{TiO}_{2}$ nanoparticles to enhance the hemocompatibility of AgNPs. After being deposited onto the PU surface, the photofunctionalized $\mathrm{Ag} @ \mathrm{TiO}_{2} \mathrm{NPs}$ coating showed excellent antibacterial properties against both Gram-positive/Gram-negative bacteria. Besides, In vitro and ex-vivo experiments demonstrated that the photo-functionalized $\mathrm{Ag}_{\mathrm{T}} \mathrm{TiO}_{2} \mathrm{NPs}$ coating had desirable hemocompatibility. This modification strategy can provide a new solution idea to improve the hemocompatibility of metal nanoparticles.

Keywords: Silver nanoparticles (AgNPs), Titanium dioxide $\left(\mathrm{TiO}_{2}\right)$, hemocompatibility, antibacterial ability, UV treatment

\section{INTRODUCTION}

Silver has historically been a commonly used antibacterial material. When silver is oxidized, the free $\mathrm{Ag}^{+}$released can act as an antibacterial/sterilizing agent by damaging the cell walls of bacteria and entering the bacteria to disrupt their metabolism and proliferation (Möhler et al., 2018). In the new century, various silver nanoparticles (AgNPs) have been developed and widely used in response to new needs. Due to their high specific surface area, AgNPs can release $\mathrm{Ag}^{+}$efficiently and stably. Also, AgNPs with nanometer size can be directly uptaken by bacteria and thus combine with thiolcontaining subcellular structures to kill bacteria synergistically with $\mathrm{Ag}^{+}$(Zheng et al., 2018).

Due to their broad-spectrum and efficient antibacterial properties, AgNPs have been used in various medical devices and medical materials, such as burn dressing, catheter, and bone cement (Chaloupka et al., 2010). However, reports of AgNPs in blood contact devices are few because of the controversy of AgNPs' hemocompatibility. Many studies have shown that AgNPs can cause adverse 
hematological events such as platelet adhesion and thrombosis when exposed to blood (Huang et al., 2016; Tran et al., 2022). This deficiency undoubtedly limits the use of AgNPs in blood-contact devices with antimicrobial needs, such as extracorporeal circuits and indwelling medical devices.

Researchers have tried many methods to improve the hemocompatibility of AgNPs. These approaches can be roughly classified into two categories. One is to construct a barrier layer to avoid direct contact between AgNPs and blood cells by utilizing biopolymer coatings or hydrogels (Fischer et al., 2015; Marulasiddeshwara et al., 2017). The other category involves co-immobilizing AgNPs with anticoagulant biomolecules to enhance hemocompatibility (Le Thi et al., 2017; Wu et al., 2020). However, both strategies are often cumbersome to prepare and do not address the hemocompatibility issue of AgNPs themselves. When the protective layer fails or AgNPs detach from the anticoagulant molecules, the AgNPs will face adverse hematological events again. Therefore, it is important to find a convenient and reliable improvement method to solve the hemocompatibility problem of AgNPs themselves.

$\mathrm{TiO}_{2}$ owns excellent biosafety and has a wide range of applications in the medical field (Jafari et al., 2020). Due to its unique photocatalytic activity, when $\mathrm{TiO}_{2}$ is subjected to UV irradiation, free radicals are generated, which can interact with the surrounding environment, thus making $\mathrm{TiO}_{2}$ exhibit biological activity (Ziental et al., 2020). Therefore, this photoinduced bioactivity of $\mathrm{TiO}_{2}$ has become a hot research topic in the last decade. The preliminary study of our team found that $\mathrm{TiO}_{2}$ after UV irradiation can acquire excellent anticoagulant properties. The changes in the physicochemical properties of the $\mathrm{TiO}_{2}$ surface, which are triggered by the $\mathrm{TiO}_{2}$ photogenerated free radicals, are thought to be responsible for this photo-induced anticoagulant properties (Chen et al., 2014). More importantly, we further revealed that this photo-induced anticoagulant properties could spread to the surface of silicon adjacent to $\mathrm{TiO}_{2}$ due to the diffusion effect of free radicals (Chen et al., 2015).

The mentioned understanding of AgNPs and $\mathrm{TiO}_{2}$ inspired us that it is possible to improve the hemocompatibility of AgNPs by utilizing the diffusible photo-induced anticoagulant properties of $\mathrm{TiO}_{2}$. To realize this idea, in this study, we firstly performed a short time of UV irradiation in seconds, using the $\mathrm{TiO}_{2}$ 's photocatalytic reduction property to load AgNPs onto $\mathrm{TiO}_{2}$ nanoparticles $\left(\mathrm{TiO}_{2} \mathrm{NPs}\right.$ ) (Chen et al., 2021). Subsequently, we performed a one-hour UV irradiation to activate the photo-induced anticoagulant properties of $\mathrm{TiO}_{2}$. By the diffusion effect of the photoinduced anticoagulant properties, the hemocompatibility of AgNPs loaded on the $\mathrm{TiO}_{2} \mathrm{NPs}$ nanoparticles was enhanced. Finally, we obtained the photo-functionalized composite nanoparticles (UV-Ag@ $\mathrm{TiO}_{2} \mathrm{NPs}$ ) with anticoagulation and antibacterial properties.

In this study, microscopic appearance and elemental analysis by transmission electron microscopy (TEM) and energy dispersive spectroscopy (EDS) were used to examine the preparation of $\mathrm{Ag@TiO}{ }_{2} \mathrm{NPs}$. Water contact angle (WCA),

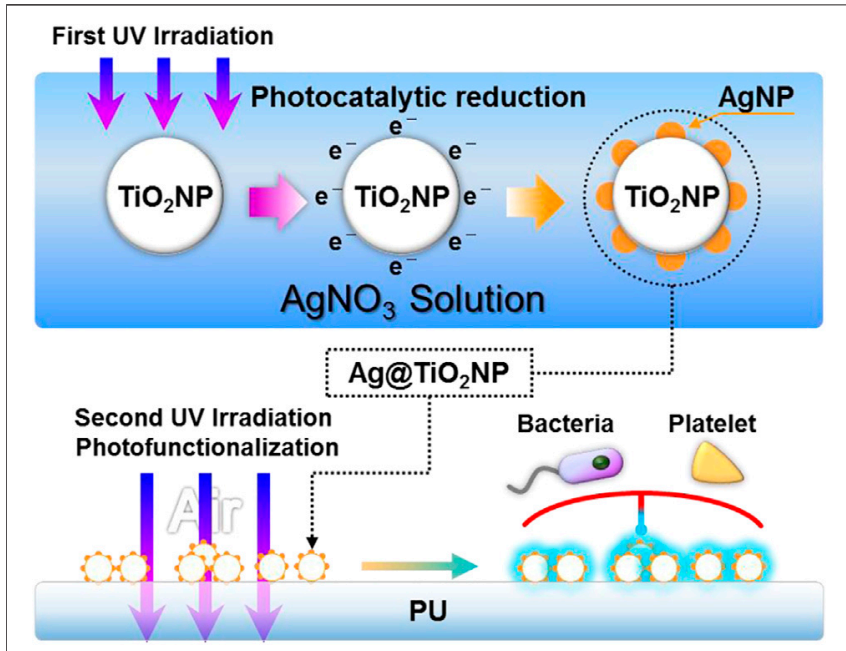

FIGURE 1 | Schematic diagram of the preparation and photofunctionalization of composite nanoparticles $\mathrm{Ag} @ \mathrm{TiO}_{2} \mathrm{NPs}$.

photocatalytic degradation of methylene blue and silver ion release experiments were used to characterize the physicochemical properties of nanoparticles coating. Grampositive Staphylococcus aureus and Gram-negative Pseudomonas aeruginosa were used to examine the antibacterial ability of photo-functionalized composite nanoparticles. In vitro platelet adhesion assay and ex-vivo antithrombogenicity test were used to examine the hemocompatibility of the photo-functionalized composite nanoparticles.

\section{MATERIALS AND METHODS}

\subsection{Preparation of ${\mathrm{Ag} @ \mathrm{TiO}_{2} \mathrm{NPs}}$}

The composite nanoparticles $\left(\mathrm{Ag} @ \mathrm{TiO}_{2} \mathrm{NPs}\right)$ were obtained by loading AgNPs particles on the surface of $\mathrm{TiO}_{2} \mathrm{NPs}$ using photocatalytic reduction, as shown in Figure 1. Briefly, P25TiO2NPs (Sigma, United States) were ultrasonically dispersed in $5 \%$ alcohol solution to obtain a suspension of $1 \mathrm{mg} / \mathrm{ml}$, and then the $\mathrm{TiO}_{2} \mathrm{NPs}$ suspension was mixed with $1 \mathrm{mg} / \mathrm{ml}$ of silver nitrate solution in equal volume. The $\mathrm{AgNO}_{3} \& \mathrm{TiO}_{2}$ suspensions were irradiated with UV light (UV light intensity $=10 \mathrm{~mW} / \mathrm{cm}^{2}$, wavelength $\lambda=365 \mathrm{~nm}$ ) at $2 \mathrm{~mm}$ liquid depth to obtain the $\mathrm{Ag} @ \mathrm{TiO}_{2} \mathrm{NPs}$ suspensions. Then the suspension was centrifuged, washed, and dried at $60^{\circ} \mathrm{C}$, obtaining $\mathrm{Ag@} \mathrm{TiO}_{2} \mathrm{NPs}$ Photocatalytic reduction times were $1,5,20$, and $60 \mathrm{~s}$ to obtain the composite nanoparticles labeled as "1\#”, “2\#”, “3\#”, and "4\#". Samples storage away from light.

\subsection{Preparation and Photo-Functionalization of ${\mathrm{Ag} @ \mathrm{TiO}_{2} \mathrm{NPs}}$ Coating}

Ultrasonically resuspend $\mathrm{Ag} @ \mathrm{TiO}_{2} \mathrm{NPs}$ with 5\% alcohol in the same volume as the Ag@ $\mathrm{TiO}_{2} \mathrm{NPs}$ suspensions in Section 2.1. 
subsequently, $1 \times 1 \mathrm{~cm}^{2}$ PU sheets were immersed in the suspension and deposited for 3 hours at room temperature to form a coating. Medical-grade silicone rubber (SR) catheters were peristaltic pump circulating with $\mathrm{Ag} @ \mathrm{TiO}_{2} \mathrm{NPs}$ suspension for $12 \mathrm{~h}$ to obtain the coating for the ex-vivo antithrombogenicity test in Section 2.5.2. After coating, samples were rinsed with RO water, dried under nitrogen, and stored away from light.

Photo-functionalization of the samples was achieved by UV irradiation (UV light intensity $=10 \mathrm{~mW} / \mathrm{cm}^{2}$, wavelength $\lambda=$ $365 \mathrm{~nm}$ ) for $1 \mathrm{~h}$.

The untreated coatings of $\mathrm{TiO}_{2}, 1 \#, 2 \#, 3 \#$, and 4\# nanoparticles are labeled as "UNT- $\mathrm{TiO}_{2} \mathrm{C}$ ", "UNT-1\#C", "UNT-2\#C", "UNT-3\#C", "UNT-4\#C", and the corresponding UV photo-functionalized nanoparticles coatings are labeled as “UV-TiO 2 C", " UV-1\#C", " UV-2\#C", " UV-3\#C", “UV-4\#C”.

\subsection{Physicochemical Characterization of $\mathrm{Ag} @ \mathrm{TiO}_{2} \mathrm{NPs}$ and $\mathrm{Ag} @ \mathrm{TiO}_{2} \mathrm{NPs}$ Coating}

The microstructure and the element distribution of $\mathrm{Ag@TiO}{ }_{2} \mathrm{NPs}$ were examined by field-emission transmission electron microscopy (TEM, JEM-2100F, JEOL, Japan) and the energy dispersive spectroscopy (EDS, JEM-2100F, JEOL, Japan).

Water contact angles (WCA) of $\mathrm{Ag@TiO} \mathrm{NPs}_{2}$ coatings were obtained by analyzing images using ImageJ software (National Institutes of Health, United States).

Photocatalytic degradation of methylene blue assay was performed to examine the photocatalytic activity of Ag@ $\mathrm{TiO}_{2} \mathrm{NPs}$ coating. In brief, methylene blue powder was prepared as a $5 \mathrm{mg} / \mathrm{L}$ solution in deionized water. A sample was immersed in $1 \mathrm{ml}$ of the solution, and UV irradiated (UV light intensity $=10 \mathrm{~mW} / \mathrm{cm}^{2}$, wavelength $\lambda=365 \mathrm{~nm}$ ) for 1,3 , and 5 h. $200 \mu \mathrm{L}$ of the solution was collected at each time point, and the absorbance (A) at a wavelength of $664 \mathrm{~nm}$ was determined using a microplate reader (BIO-TEK Instruments, United States). The relationship between $\mathrm{A}$ and the degradation rate $(\mathrm{G})$ was calculated as

$$
\mathrm{G}=\left[\left(\mathrm{A}_{0}-\mathrm{A}_{\mathrm{t}}\right) / \mathrm{A}_{0}\right] \times 100 \%
$$

where $A_{0}$ is the original absorbance of the undegraded methylene blue, and $A_{t}$ is the absorbance value after thours of degradation.

In the $\mathrm{Ag}^{+}$release test, atomic absorption spectroscopy (AAS, TAS-990F, Beijing Purkinje General Instrument Co., Ltd., China) was performed to measure the release of $\mathrm{Ag}^{+}$from different samples into phosphate-buffered saline (PBS; $\mathrm{pH}=7.4$ ). Briefly, PU samples coated with Ag@ $\mathrm{TiO}_{2} \mathrm{NPs}$ were immersed in $1 \mathrm{ml}$ of PBS in the dark. The PBS was collected and replaced with $1 \mathrm{ml}$ of fresh PBS every 2 days. This process was repeated for a total of 14 days. All collected PBS solutions were analyzed for their content of $\mathrm{Ag}^{+}$, and an $\mathrm{Ag}^{+}$time-release curve was plotted.

\subsection{Antibacterial Assay}

In this study, Staphylococcus aureus (S. aureus) and Pseudomonas aeruginosa ( $P$. aeruginosa) were used to evaluate the antibacterial ability of the Ag@ $\mathrm{TiO}_{2} \mathrm{NPs}$ coating. Briefly, S. aureus and $P$. aeruginosa obtained from Sichuan Provincial People's Hospital were inoculated onto blood agar plates and cultivated in an incubator at $37^{\circ} \mathrm{C}$. After the appearance of multiple colonies, $S$. aureus and $P$. aeruginosa were collected and dispersed in an F12 medium containing $10 \%$ fetal bovine serum (FBS, Sigma, United States). The density of $S$. aureus and $P$. aeruginosa was adjusted to $1 \times 10^{6}$ colony forming units $(\mathrm{CFU}) / \mathrm{mL}$, and then $1 \mathrm{ml}$ of bacterial suspension was added to the wells of a 24-well plate to soak the samples. After incubation at $37^{\circ} \mathrm{C}$ for 6 hours, the samples were washed three times and then transferred to a new 24-well plate containing a mixture of F12 FBS medium and cell counting kit-8(CCK-8, APExBIO Ltd., Houston, United States) agent. After $3 \mathrm{~h}$ of incubation, the activity of adherent bacteria was tested by detecting the absorbance of the medium at a wavelength of $450 \mathrm{~nm}$ using a microplate reader. The samples were then fixed with $2.5 \%$ glutaraldehyde for $12 \mathrm{~h}$, after which they were dehydrated, and the adherent bacteria on the samples were observed and analyzed with an optical microscope (OM, DM4000M; Leica, Germany).

\subsection{Evaluation of Hemocompatibility 2.5.1 Platelet Static Adhesion Test}

Fresh whole blood was drawn from human healthy adult volunteers and anticoagulated with citric acid dextrose (ACD) (blood to ACD ratio of 9:1), in compliance with the ethical standards of Southwest Jiaotong University. Then, the fresh ACD blood was centrifuged at 1,500 rpm for $15 \mathrm{~min}$, and thus platelet-rich plasma (PRP) was obtained. The PU samples $(1 \times$ $1 \mathrm{~cm}^{2}$ ) deposited with the nanoparticles coatings were placed in 24 -well cell culture plates and incubated with PRP $(100 \mu \mathrm{L}$ per sample) at $37^{\circ} \mathrm{C}$ for 1 hour under static conditions. After incubation, the samples were rinsed carefully three times with $0.9 \%$ saline to remove non-adherent platelets. Subsequently, the samples were fixed with $2.5 \%$ glutaraldehyde for 2 hours at room temperature. After typical rhodamine staining (Sigma, United States), the number of platelet adhesions in each sample was calculated by Image J software with six random fluorescence microscopy (DMRX, Leica, Germany) images $($ size $=400 \times)$. Furthermore, the morphology of the adhered platelets was observed under a scanning electron microscope (SEM, Quanta 200; FEI, Holland).

\subsubsection{Antithrombogenicity Test by Ex-Vivo Blood Circulation}

All succeeding procedures were performed in compliance with the China Council on Animal Care and Southwest Jiaotong University Animal Use protocol, following all the ethical guidelines for experimental animals.

The establishment of ex-vivo blood circulation has been described elsewhere (Qiu et al., 2021). Thrombogenicity of the SR catheters was assessed using an ex-vivo arteriovenous (AV) shunt model in the rabbit. For the detail of the AV shunt model, a custom-built extracorporeal circulation (ECC) pipeline with three parallel channels was set up. The ECC pipeline was made of medical-grade polyvinyl chloride (PVC) tubing. Samples, including the uncoated SR, UNT-1\#C, and UV-1\#C catheters, were connected to the ECC system. 


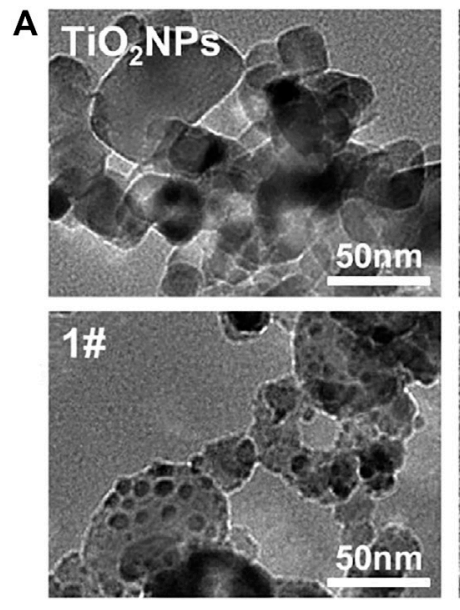

C

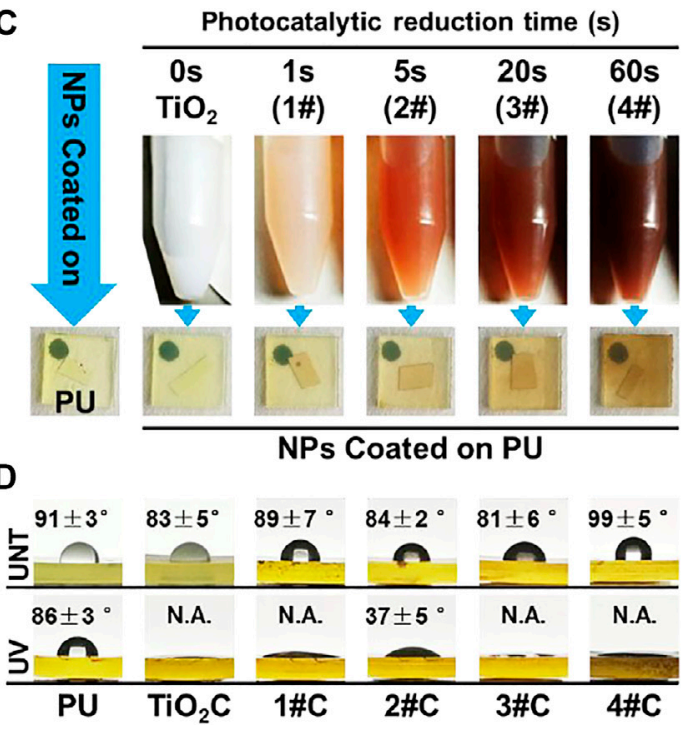

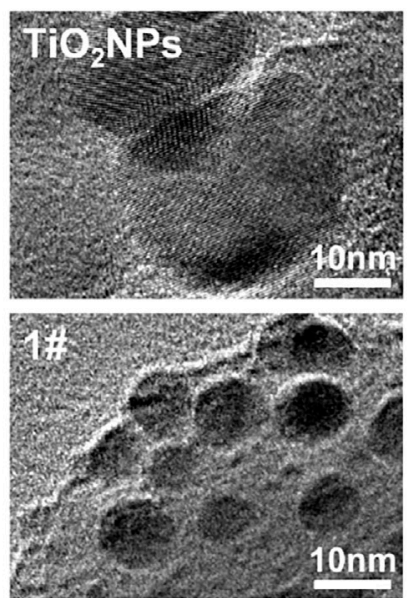

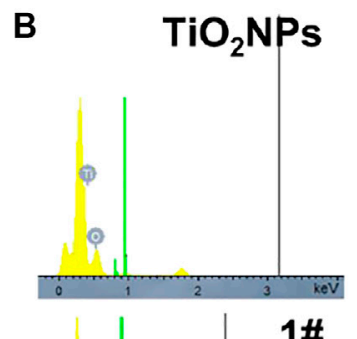

E

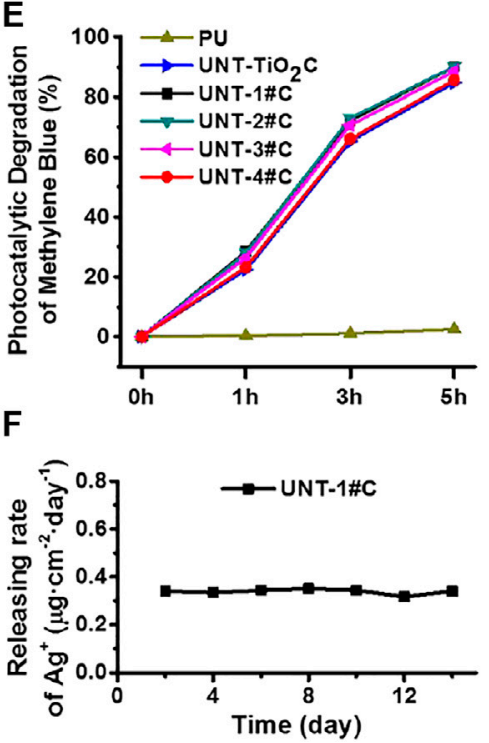

FIGURE 2|TEM microscopic images of $\mathrm{TiO}_{2} \mathrm{NPs}$ before and after photocatalytic reduction in silver nitrate solution (A) and the results of EDS elemental analysis (B); photos of $\mathrm{Ag} @ \mathrm{TiO}_{2} \mathrm{NPs}$ suspensions and corresponding coating on PU sheets at different photocatalytic reduction times (C); WCA of Ag@TiO ${ }_{2} \mathrm{NPs}$ coatings on PU

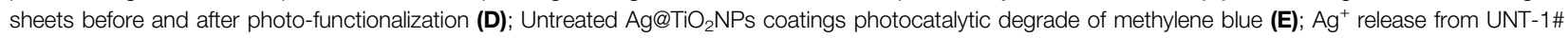
coating (F).

Rabbits (New Zealand white rabbits, $2.5 \mathrm{~kg}$ ) were anesthetized by intravenous injection of sodium pentobarbital $(30 \mathrm{mg} / \mathrm{kg})$. The rabbit left carotid artery and the right external jugular vein were isolated through a midline neck incision. The AV custom-built extracorporeal circulation (ECC) was placed into position by cannulating the left carotid artery for ECC inflow and the right external jugular vein for ECC outflow (Figure 5A). The flow through the ECC was started by unclamping the arterial and venous sides of the ECC. Animals had no systemic anticoagulation throughout the experiment.

After 30min on ECC, the circuits were clamped, removed from the animal, rinsed with $0.9 \%$ saline $(\mathrm{pH} 7.4)$ gently, and drained. The cross-sections of the catheters were photographed for determination of the occlusion rates. Then, the residual thrombosis in the catheters was fixed in $2.5 \%$ glutaraldehyde solution overnight at room temperature and dehydrated. After weighing, samples undergo a micromorphological analysis by scanning electron microscopy (SEM, Quanta 200, FEI, Holland).

\section{RESULTS AND DISCUSSION}

\subsection{Characterization of $\mathrm{Ag} @ \mathrm{TiO}_{2} \mathrm{NPs}$}

To confirm that Ag@ $\mathrm{TiO}_{2} \mathrm{NPs}$ were successfully prepared, TEM was used to characterize the microstructure of the nanoparticles, and EDS was used to perform elemental analysis of the nanoparticles. From the TEM results, additional spherical secondary structures with diameters of $5-10 \mathrm{~nm}$ were observed on the surface of $1 \#$ nanoparticles compared to $\mathrm{TiO}_{2} \mathrm{NPs}$ (Figure 2A). the signal of elemental $\mathrm{Ag}$ appeared in the EDS data for 1\# nanoparticles (Figure 2B), which indicates that the spherical secondary structures appearing on the surface of $1 \#$ nanoparticles are 

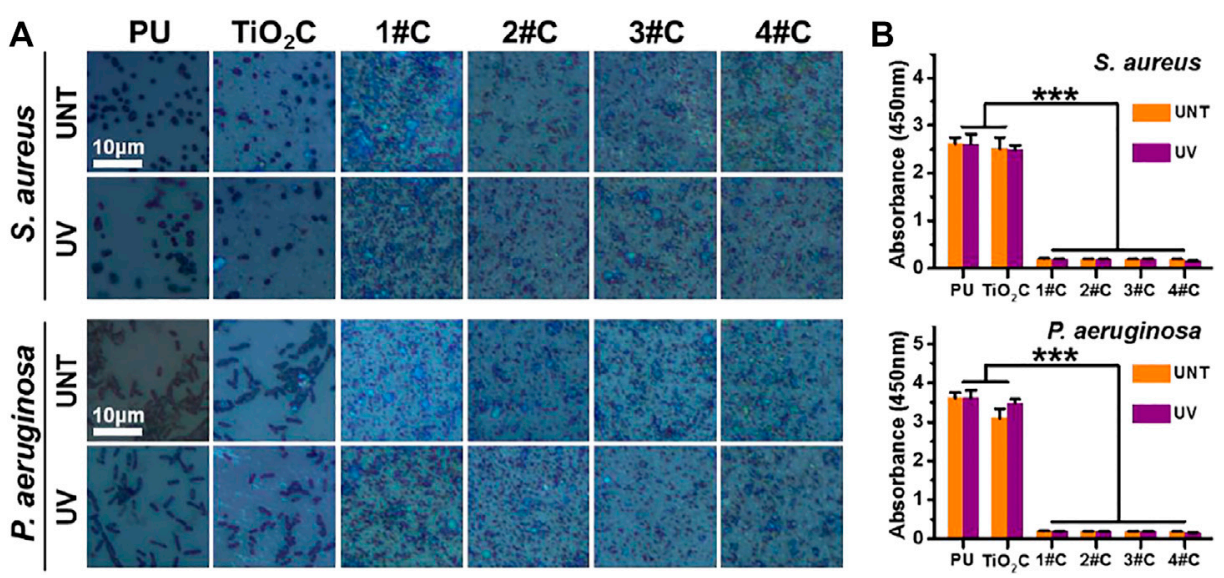

FIGURE 3 | Antibacterial activity assay. Optical microscopy images of bacteria cultured on nanoparticles coatings before and after photo-functionalization (A) and bacterial activity measured by CCK-8 kit (B).

AgNPs. These AgNPs should be the products of $\mathrm{Ag}^{+}$reduction by electrons generated on the surface of $\mathrm{TiO}_{2} \mathrm{NPs}$ during the photocatalytic reduction process (Lu et al., 2013; Chen et al., 2021).

The results of TEM and EDS indicate that $\mathrm{Ag@TiO}{ }_{2} \mathrm{NPs}$ were successfully prepared. Furthermore, the color of the suspensions of $\mathrm{Ag} @ \mathrm{TiO}_{2} \mathrm{NPs}$ deepened with the prolongation of the photocatalytic reduction time, which implied that the AgNPs content in Ag@ $\mathrm{TiO}_{2} \mathrm{NPs}$ might increase with the photocatalytic reduction time prolongation ( $\mathrm{Lu}$ et al., 2013). The color of the coatings deposited by $\mathrm{Ag} @ \mathrm{TiO}_{2} \mathrm{NPs}$ onto the PU sheets also showed the same trend (Figure 2C).

\subsection{Characterization of ${\mathrm{Ag} @ \mathrm{TiO}_{2} \mathrm{NPs}}$ Coating}

The photo-induced hydrophilicity of $\mathrm{TiO}_{2}$ has been reported in many pieces of literature (Carp et al., 2004). Excellent hydrophilicity is considered to facilitate biofouling resistance (He et al., 2021). Compared with the non-photo-functionalized UNT-Ag@ $\mathrm{TiO}_{2} \mathrm{NPs}$ coatings, the photo-functionalized UV-Ag@ $\mathrm{TiO}_{2} \mathrm{NPs}$ coatings showed a significant decrease in WCA and exhibited strong photo-induced hydrophilicity (Figure 2D), which means that photo-functionalized nanoparticle coatings may be somehow more anticoagulant and antibacterial than their counterparts in the group. Among all UV-Ag@TiO ${ }_{2} \mathrm{NPs}$ coatings, 2\# coating had the highest water contact angle, perhaps related to the content of AgNPs on the surface of $\mathrm{Ag} @ \mathrm{TiO}_{2} \mathrm{NPs}$, the exact mechanism of which needs to be further investigated.

The photocatalytic oxidation activity of $\mathrm{TiO}_{2}$ is another manifestation of the photocatalytic activity of $\mathrm{TiO}_{2}$, which is closely related to the mechanism of photo-induced anticoagulant properties of $\mathrm{TiO}_{2}$ (Chen et al., 2014; Chen et al., 2015). Although it has been reported in the literature that loading AgNPs on the surface of $\mathrm{TiO}_{2}$ can enhance the photocatalytic activity of $\mathrm{TiO}_{2}$ by forming Schottky energy barriers (Gomathi Devi and Kavitha, 2016). However, in this study, through the photocatalytic oxidation decomposition of methylene blue, we found that the loading of AgNPs did not significantly affect the photocatalytic oxidation activity of Ag@ $\mathrm{TiO}_{2} \mathrm{NPs}$ coating, compared to $\mathrm{TiO}_{2} \mathrm{NPs}$ coating (Figure 2E).
$\mathrm{The}^{+} \mathrm{g}^{+}$release rate is closely related to AgNPs' biosafety and antimicrobial properties (Marchioni et al., 2018). 1\# nanoparticles were used to analyze their $\mathrm{Ag}^{+}$release in PBS after coating on $\mathrm{PU}$, and it was found that the $\mathrm{Ag}^{+}$release rate of UNT-1\#C was stable around $0.35 \mu \mathrm{g} \mathrm{cm}^{-2} \mathrm{day}^{-1}$ (Figure 2F), which was considered to be safe (Liu et al., 2018).

\subsection{Analysis of Antibacterial Property}

Here, Gram-positive Staphylococcus aureus (S. aureus) and Gramnegative Pseudomonas aeruginosa ( $P$. aeruginosa) were used to examine the broad-spectrum antimicrobial resistance of the Ag@ $\mathrm{TiO}_{2} \mathrm{NPs}$ coatings. The results of optical microscopy showed that morphologically intact $S$. aureus and $P$. aeruginosa could be seen on the surface of all $\mathrm{PU}$ and $\mathrm{TiO}_{2} \mathrm{NPs}$ coatings, in contrast to all $\mathrm{Ag} @ \mathrm{TiO}_{2} \mathrm{NPs}$ coatings with a large amount of debris, probably necrotic bacterial fragments (Figure 3A).

The results of the cellular activity analysis (Figure 3B) were consistent with the observation under optical microscopy, where the bacterial activity on the surface of the $\mathrm{TiO}_{2} \mathrm{NPs}$ coating was similar to that of the PU. In contrast, all Ag@ $\mathrm{TiO}_{2} \mathrm{NPs}_{\text {coatings }}$ exhibited significant antimicrobial activity compared to the PU substrate and the $\mathrm{TiO}_{2} \mathrm{NPs}$ coating. Notably, there was no significant difference between the antimicrobial properties of UNT-Ag@TiO ${ }_{2} \mathrm{NPs}$ coatings and UV-Ag@ $\mathrm{TiO}_{2} \mathrm{NPs}$ coatings, suggesting that the photo-functionalization treatment did not affect the AgNPs portion of $\mathrm{Ag}_{\mathrm{TiO}} \mathrm{NPs}_{2}$ to perform the antimicrobial function. Another noteworthy point is that the antimicrobial performance of $1 \#$ coatings with the lowest AgNPs loading is comparable to that of $4 \#$ coaitngs with the highest AgNPs loading, indicating a powerful antimicrobial performance.

\subsection{Analysis of Anticoagulant Property in vitro}

In vitro platelet adhesion experiments were performed using deposited Ag@ $\mathrm{TiO}_{2} \mathrm{NPs}$ coatings on PU sheets, for PU is widely used in various blood contact devices. The results of fluorescence images (Figure 4A) and adhesion density 

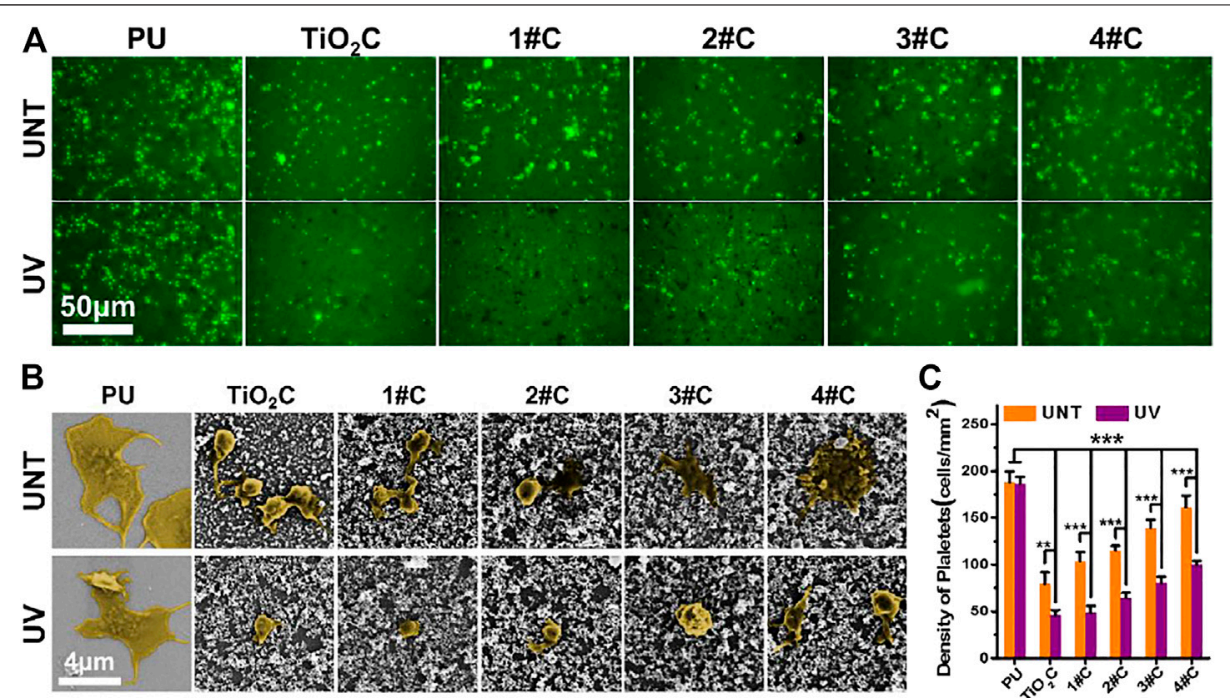

FIGURE 4 | In vitro platelet adhesion assay. Fluorescence images (A), SEM images (B), and adhesion density (C) of adhered platelet on nanoparticles coatings.
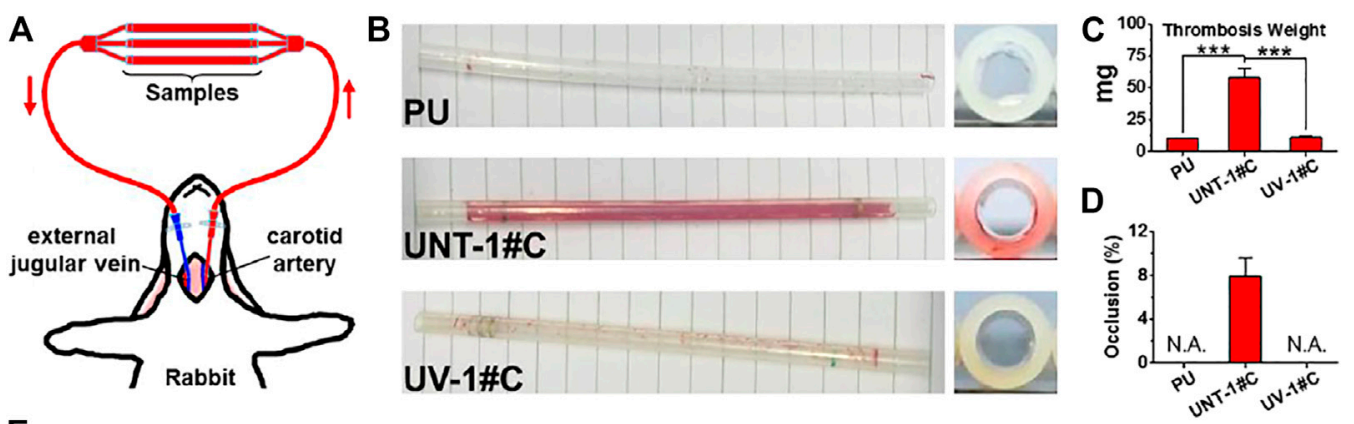

$\mathbf{E}$
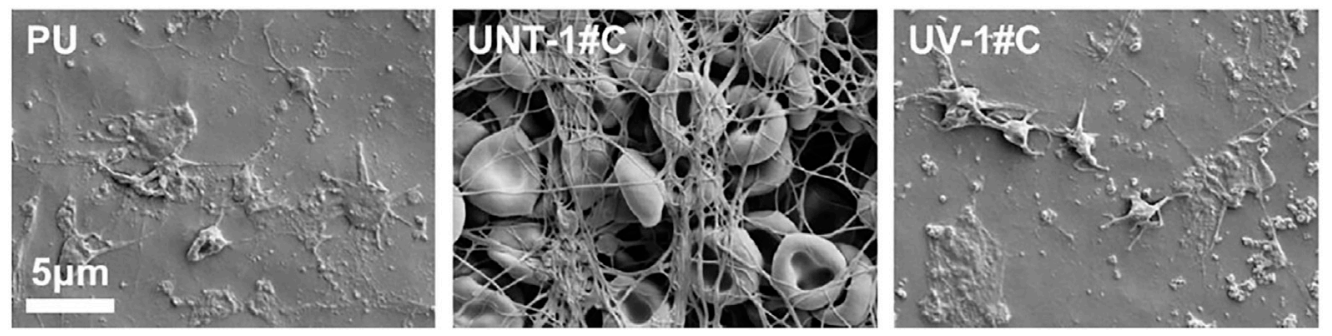

FIGURE 5 | Schematic diagram of the ex-vivo blood circulation experiment (A); photograph of the catheter after the experiment (B); the weight of the thrombus formed in the catheter (C); catheter occlusion rate (D); microscopic image of the catheter lining by SEM (E).

(Figure 4C) showed that all nanoparticles coatings significantly reduced the number of platelet adhesions after photofunctionalization compared with that before treatment. Besides, the platelet adhesions density on the photofunctionalized nanoparticle coatings was significantly lower than that of PU substrates. Notably, the platelet adhesion density of nanoparticles coatings tended to increase with the prolongation of photocatalytic reduction time in the composite nanoparticles preparation stage. Considering the issue of blood compatibility of AgNPs, this result is similar to the phenomenon that the color of Ag@TiO2NPs suspension deepens with increasing photocatalytic reduction time (Figure 2C), supporting the idea that the AgNPs content in Ag@TiO2NPs increases with increasing photocatalytic reduction time.The SEM results of platelet adhesion (Figure $4 \mathrm{~B}$ ) showed that the platelets on the PU substrate were in a partially spreading dendrites state, with the degree of spread much higher than that of platelets on the UV-photo-functionalized nanoparticles coating, which was between spherical to partially spread. In contrast, the degree of platelet spreading on all nanoparticles coatings showed a 
tendency to increase with increasing photocatalytic reduction time at the composite nanoparticles preparation stage. This trend is consistent with the results of platelet adhesion density, which could be similarly influenced by the content of AgNPs in the composite nanoparticles.

The results of platelet adhesion experiments suggested that the $\mathrm{Ag@TiO} \mathrm{NPs}_{2}$ coatings can acquire anti-platelet adhesion ability after photo-functionalization. However, this photo-induced anticoagulant properties of the UV-Ag@TiO $\mathrm{NPs}_{2}$ may be weakened by increasing the content of AgNPs, this implies that the photo-induced anticoagulant properties of $\mathrm{TiO}_{2}$ have a limited or dose-dependent improvement on the hemocompatibility of AgNPs. Therefore, proper loading of AgNPs is essential to impart UV-Ag@TiO2NPs with excellent antimicrobial properties without causing deterioration of hemocompatibility.

Here, combined with the results of the antimicrobial experiments and in vitro platelet adhesion assays, the UV-1\# sample with the lowest silver loading may be an ideal candidate capable of obtaining balanced antimicrobial and anticoagulant properties.

\subsection{Ex-vivo Antithrombogenicity Test}

The Antithrombogenicity test by ex-vivo blood circulation provides a more comprehensive test of the anticoagulant capacity of the UV-Ag@ $\mathrm{TiO}_{2} \mathrm{NPs}$ coating compared to the in vitro blood test. SR is a material commonly used for central venous catheters, which is a typical medical device that requires both anticoagulation and antimicrobial activity. Considering the in vitro platelet adhesion and Antibacterial Assay results, SR catheters deposited with 1 \# nanoparticles were selected for the exvivo blood circulation assay. After $30 \mathrm{~min}$ of ex-vivo blood circulation, the surface of the UNT-1\# coating showed patches of sizable thrombus layer that blocked nearly $8 \%$ of the catheter lumen compared to the UV-1\# coating with only a small amount of thrombus on the surface (Figures 5B,D). The analysis of the weight of the formed thrombus showed a significant increase in UNT-1\# coating compared to both UV-1\# coating and PU substrate (Figure 5C). At the same time, there was no significant difference in $\mathrm{UV}-1 \#$ coating compared to $\mathrm{PU}$ substrate.

SEM images of the inner wall of the catheter revealed that UNT-1\# coating had fully initiated the coagulation mechanism, and the fibrinogen network captured a large number of red blood cells, forming a thrombus layer that completely covered the substrate. In contrast, the UV-1\# coating and the PU substrate were similar, with only some platelets adhering to the surface (Figure 5E). It is worth noting that most of the erythrocytes in the thrombus layer remain in typical form, and combined with the data on the rate of silver ion release from the coating (Figure 2F), hemolysis may not be a problem that the coating would cause.

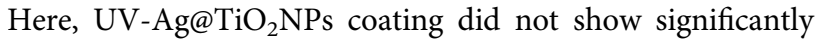
better results than PU substrates in the ex-vivo blood assay as in the in vitro platelet adhesion assay. This may be due to the dynamic environment and more comprehensive blood composition in the ex-vivo blood circulation assay (Courtney et al., 1994). Nevertheless, the results of in vitro platelet adhesion and ex-vivo blood experiments were sufficient to suggest that loading AgNPs onto the surface of $\mathrm{TiO}_{2} \mathrm{NPs}$ and performing UVPhoto-functionalization treatment was an effective way to improve their hemocompatibility.

\section{CONCLUSION}

This study prepared a composite nanoparticles $\left(\mathrm{Ag} @ \mathrm{TiO}_{2} \mathrm{NPs}\right)$ by loading AgNPs onto $\mathrm{TiO}_{2} \mathrm{NPs}$ using a photocatalytic reduction method. Then, we utilized secondary UV irradiation to photofunctionalize Ag@ $\mathrm{TiO}_{2} \mathrm{NPs}$ to improve the hemocompatibility of $\mathrm{TiO}_{2} \mathrm{NPs}$ and AgNPs. In vitro and ex-vivo experiments showed that such photo-functionalized UV-Ag@TiO ${ }_{2} \mathrm{NPs}_{\text {coatings are }}$ endowed with excellent hemocompatibility. When UV-Ag@ $\mathrm{TiO}_{2} \mathrm{NPs}$ were deposited onto the $\mathrm{PU}$ and SR to form a coating, they significantly inhibited platelet adhesion and activation, showing anticoagulant properties not inferior to those of medical-grade substrates. In addition, this UV-Ag@ $\mathrm{TiO}_{2} \mathrm{NPs}$ coating exhibited excellent antibacterial properties against both Gram-positive and Gram-negative bacteria. Therefore, these UV-Ag@ $\mathrm{TiO}_{2} \mathrm{NPs}$ could service the medical devices that require both anticoagulant and antibacterial properties, such as central venous catheters. More importantly, this UV-photo-functionalized modification strategy relying on $\mathrm{TiO}_{2}$ could provide a new idea to solve the problem of blood compatibility of functionalized metal nanoparticles similar to AgNPs.

\section{DATA AVAILABILITY STATEMENT}

The original contributions presented in the study are included in the article/Supplementary Material, further inquiries can be directed to the corresponding authors.

\section{ETHICS STATEMENT}

The animal study was reviewed and approved by Ethics Committee of Southwest Jiaotong University.

\section{AUTHOR CONTRIBUTIONS}

XC: Experiment, Writing- Original draft preparation. SD, LL, PL, and PEY: Experiment, Original draft preparation. YL and AZ: Data curation and Writing- Reviewing. PIY: Supervision and language polishment. $\mathrm{NH}$ and JC: Conceptualization and manuscript revision.

\section{FUNDING}

This work was supported by the National Natural Science Foundation of China (No. 31870958 (PIY), 31700821 (JC), 81771988 (AZ), and the Sichuan Science and Technology Program (No.20GJHZ0268) (AZ). 


\section{REFERENCES}

Carp, O., Huisman, C. L., and Reller, A. (2004). Photoinduced Reactivity of Titanium Dioxide. Prog. Solid State Chem. 32 (1-2), 33-177. doi:10.1016/j. progsolidstchem.2004.08.001

Chaloupka, K., Malam, Y., and Seifalian, A. M. (2010). Nanosilver as a New Generation of Nanoproduct in Biomedical Applications. Trends Biotechnol. 28 (11), 580-588. doi:10.1016/j.tibtech.2010.07.006

Chen, J., Zhao, A., Chen, H., Liao, Y., Yang, P., Sun, H., et al. (2014). The Effect of Full/partial UV-Irradiation of $\mathrm{TiO} 2$ Films on Altering the Behavior of Fibrinogen and Platelets. Colloids Surf. B: Biointerfaces 122, 709-718. doi:10. 1016/j.colsurfb.2014.08.004

Chen, J., Yang, P., Liao, Y., Wang, J., Chen, H., Sun, H., et al. (2015). Effect of the Duration of UV Irradiation on the Anticoagulant Properties of Titanium Dioxide Films. ACS Appl. Mater. Inter. 7 (7), 4423-4432. doi:10.1021/ am509006y

Chen, J., Dai, S., Liu, L., Maitz, M. F., Liao, Y., Cui, J., et al. (2021). Photofunctionalized $\mathrm{TiO} 2$ Nanotubes Decorated with Multifunctional Ag Nanoparticles for Enhanced Vascular Biocompatibility. Bioactive Mater. 6 (1), 45-54. doi:10.1016/j.bioactmat.2020.07.009

Courtney, J. M., Lamba, N. M. K., Sundaram, S., and Forbes, C. D. (1994). Biomaterials for Blood-Contacting Applications. Biomaterials 15 (10), 737-744. doi:10.1016/0142-9612(94)90026-4

Fischer, M., Vahdatzadeh, M., Konradi, R., Friedrichs, J., Maitz, M. F., Freudenberg, U., et al. (2015). Multilayer Hydrogel Coatings to Combine Hemocompatibility and Antimicrobial Activity. Biomaterials 56, 198-205. doi:10.1016/j.biomaterials.2015.03.056

Gomathi Devi, L., and Kavitha, R. (2016). A Review on Plasmonic metal $\square \mathrm{TiO} 2$ Composite for Generation, Trapping, Storing and Dynamic Vectorial Transfer of Photogenerated Electrons across the Schottky junction in a Photocatalytic System. Appl. Surf. Sci. 360, 601-622. doi:10.1016/j.apsusc.2015.11.016

He, Z., Yang, X., Wang, N., Mu, L., Pan, J., Lan, X., et al. (2021). Anti-Biofouling Polymers with Special Surface Wettability for Biomedical Applications. Front. Bioeng. Biotechnol. 9, 807357. doi:10.3389/fbioe.2021.807357

Huang, H., Lai, W., Cui, M., Liang, L., Lin, Y., Fang, Q., et al. (2016). An Evaluation of Blood Compatibility of Silver Nanoparticles. Sci. Rep. 6 (1), 1-15. doi:10. 1038/srep25518

Jafari, S., Mahyad, B., Hashemzadeh, H., Janfaza, S., Gholikhani, T., and Tayebi, L. (2020). Biomedical Applications of $\mathrm{TiO}_{2}$ Nanostructures: Recent Advances. Ijn 15, 3447-3470. doi:10.2147/ijn.s249441

Le Thi, P., Lee, Y., Kwon, H. J., Park, K. M., Lee, M. H., Park, J.-C., et al. (2017). Tyrosinase-mediated Surface Coimmobilization of Heparin and Silver Nanoparticles for Antithrombotic and Antimicrobial Activities. ACS Appl. Mater. Inter. 9 (24), 20376-20384. doi:10.1021/acsami.7b02500

Liu, X., Chen, J., Qu, C., Bo, G., Jiang, L., Zhao, H., et al. (2018). A Mussel-Inspired Facile Method to Prepare Multilayer-AgNP-Loaded Contact Lens for Early Treatment of Bacterial and Fungal Keratitis. ACS Biomater. Sci. Eng. 4 (5), 1568-1579. doi:10.1021/acsbiomaterials.7b00977
Lu, Q., Lu, Z., Lu, Y., Lv, L., Ning, Y., Yu, H., et al. (2013). Photocatalytic Synthesis and Photovoltaic Application of Ag-TiO2 Nanorod Composites. Nano Lett. 13 (11), 5698-5702. doi:10.1021/nl403430x

Marchioni, M., Jouneau, P.-H., Chevallet, M., Michaud-Soret, I., and Deniaud, A. (2018). Silver Nanoparticle Fate in Mammals: Bridging In Vitro and In Vivo Studies. Coord. Chem. Rev. 364, 118-136. doi:10.1016/j.ccr.2018.03.008

Marulasiddeshwara, M. B., Dakshayani, S. S., Sharath Kumar, M. N., Chethana, R., Raghavendra Kumar, P., and Devaraja, S. (2017). Facile-one Pot-Green Synthesis, Antibacterial, Antifungal, Antioxidant and Antiplatelet Activities of Lignin Capped Silver Nanoparticles: A Promising Therapeutic Agent. Mater. Sci. Eng. C 81, 182-190. doi:10.1016/j.msec.2017.07.054

Möhler, J. S., Sim, W., Blaskovich, M. A. T., Cooper, M. A., and Ziora, Z. M. (2018). Silver Bullets: A New Lustre on an Old Antimicrobial Agent. Biotechnol. Adv. 36 (5), 1391-1411. doi:10.1016/j.biotechadv.2018.05.004

Qiu, H., Tu, Q., Gao, P., Li, X., Maitz, M. F., Xiong, K., et al. (2021). PhenolicAmine Chemistry Mediated Synergistic Modification with Polyphenols and Thrombin Inhibitor for Combating the Thrombosis and Inflammation of Cardiovascular Stents. Biomaterials 269, 120626. doi:10.1016/j.biomaterials. 2020.120626

Tran, H. D., Moonshi, S. S., Xu, Z. P., and Ta, H. T. (2022). Influence of Nanoparticles on the Haemostatic Balance: between Thrombosis and Haemorrhage. Biomater. Sci. 10 (1), 10-50. doi:10.1039/d1bm01351c

Wu, H., Su, M., Jin, H., Li, X., Wang, P., Chen, J., et al. (2020). Rutin-Loaded Silver Nanoparticles with Antithrombotic Function. Front. Bioeng. Biotechnol. 8, 1356. doi: $10.3389 /$ fbioe. 2020.598977

Zheng, K., Setyawati, M. I., Leong, D. T., and Xie, J. (2018). Antimicrobial Silver Nanomaterials. Coord. Chem. Rev. 357, 1-17. doi:10.1016/j.ccr.2017.11.019

Ziental, D., Czarczynska-Goslinska, B., Mlynarczyk, D. T., Glowacka-Sobotta, A., Stanisz, B., Goslinski, T., et al. (2020). Titanium Dioxide Nanoparticles: Prospects and Applications in Medicine. Nanomaterials 10 (2), 387. doi:10. 3390/nano10020387

Conflict of Interest: The authors declare that the research was conducted in the absence of any commercial or financial relationships that could be construed as a potential conflict of interest.

Publisher's Note: All claims expressed in this article are solely those of the authors and do not necessarily represent those of their affiliated organizations, or those of the publisher, the editors and the reviewers. Any product that may be evaluated in this article, or claim that may be made by its manufacturer, is not guaranteed or endorsed by the publisher.

Copyright (C) 2022 Chen, Dai, Liu, Liu, Ye, Liao, Zhao, Yang, Huang and Chen. This is an open-access article distributed under the terms of the Creative Commons Attribution License (CC BY). The use, distribution or reproduction in other forums is permitted, provided the original author(s) and the copyright owner(s) are credited and that the original publication in this journal is cited, in accordance with accepted academic practice. No use, distribution or reproduction is permitted which does not comply with these terms. 